\title{
Effects on Polychromatic Integral Diffraction Efficiency for Dual-wavebands Harmonic Diffractive Optical Elements
}

\author{
Xiaowei Chang ${ }^{1,}{ }^{*}$, Yi Zheng $^{1}$ \\ ${ }^{1}$ School of Science, Beijing Jiaotong University, Beijing, China \\ ${ }^{*}$ Corresponding author
}

\begin{abstract}
Keywords: optical design; harmonic diffractive optical elements; infrared optical system; diffraction efficiency; polychromatic integral diffraction efficiency.
\end{abstract}

\begin{abstract}
Harmonic diffractive optical elements(HDOEs) can make multiple-wavebands imaged on the same image plane, which have be widely used in the infrared imaging optical systems. The mathematical model of effects on diffraction efficiency and polychromatic integral diffraction efficiency(PIDE) caused by single point diamond turning processing for dual-wavebands harmonic diffractive optical elements have been set up. And the expressions on diffraction efficiency and PIDE by machining errors including micro-structure heights and widths have been deduced. In order to make the maximum PIDE value over the dual-wavebands, the machining errors should be designed and distributed. Based on the above analysis, the dual-waveband harmonic diffractive optical elements used in 3.7um-4.3um and 8.7um-8.7um wavebands have been analyzed and simulated with MATLAB software on the two conditions including the oblique incidence and general incident distributions for the machining errors, which can be directly used to evaluate broadband hybrid diffractive-reflective optical systems imaging quality. Through the relationship between PIDE and machining errors of dual-wavebands HDOEs, the optical modulation transfer function can be analyzed. The results can be applied to dual band imaging optical systems for controlling the harmonic diffractive optical element processing and machining errors as well as the incident angle design for the hybrid diffractive-reflective optical systems.
\end{abstract}

\section{Introduction}

Diffractive optical elements have special dispersion and temperature properties, which are widely used in modern imaging optical systems.[1-8] Single traditional diffractive optical elements have strong negative dispersion characteristics. In order to overcome the disadvantages of huge optical chromatic aberration of diffractive optical elements, the concept of the harmonic diffractive elements was developed in 1995. [9] Harmonic diffractive optical element is also called multistage diffractive lens, which can implement $100 \%$ diffraction efficiency in a series of separate wavelengths, obtain the same focal power, and decrease dispersion. To a certain extent, it can overcome the disadvantages of huge optical chromatic aberration of traditional diffractive optical elements. For diffractive optical elements, diffraction efficiency has a decisive effect on its use. For the diffractive optical elements in the imaging optical system, the technique of single point diamond turning is often used for machining. Therefore, it is inevitable that some machining errors will be produced to reduce the diffraction efficiency of diffracted optical elements in the diffractive hybrid optical system, resulting in the degradation of the imaging quality.

At present, the machining precision of diffractive optical elements is in a high level, but the machining errors are still inevitable. The machining errors of diffractive optical elements directly affect the diffraction efficiency, which leads to the degradation of the imaging quality of the diffractive hybrid optical system. [10] There are two main errors in the machining of diffractive optical elements: The first is the height errors of the diffractive microstructure, and the second is the cycle width errors of the diffractive microstructure. Usually, these two errors come together and work together.

At present, the machining errors type of single point diamond turning has been partially studied. The literature[11] analyzed the effects of machining errors on continuous surface diffractive optical 
elements, and the effects of machining height and cycle width errors of diffractive microstructure on the diffraction efficiency of multi-layer diffractive optical elements. The effects of machining errors on the average diffraction efficiency of the polychromatic integral was studied in the literature [12]. However, there is no research on the distribution issue of the machining errors of the dual-waveband harmonic diffractive optical elements based on the theory of the polychromatic integral average diffraction efficiency. For the imaging optical system, MTF is the imaging property of the whole band range of the optical system, which has an important relationship with the polychromatic integral average diffraction efficiency of the diffractive optical elements. This paper, based on the theory about the effects of machining errors on single-layer diffractive optical element, established a mathematical model of the polychromatic integral average diffraction efficiency of HDOEs. It also deduced the expression of the effects of machining errors on the dual-waveband harmonic diffractive optical elements. Then based on this theory, the machining errors distribution of dual-waveband harmonic diffractive optical elements was designed and controlled. The research results can be used as a guidance to control machining errors of HDOEs in the multiband refractive diffractive hybrid imaging optical system. It can also improve the design of the refractive diffractive hybrid imaging optical system.

\section{The Relationship between Machining Errors of Dual-waveband Harmonic Diffractive Optical Elements and the Polychromatic Integral Average Diffraction Efficiency}

The characteristic of HDOEs is that the optical path difference between the adjacent annuluses is the integer multiple $p(p \geq 2)$ of the design wavelength. That is to say, the height of surface microstructure of the harmonic diffractive elements in air is p times of the ordinary diffractive elements. As shown in Figure 3.1, the left one is harmonic diffractive elements $p \geq 2$, the right one is ordinary diffractive elements $p=1$.

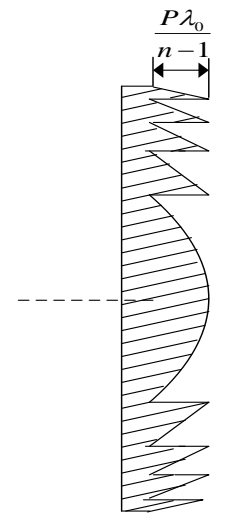

(a)

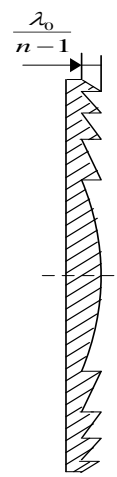

(b)

Fig.1 Structure of two kinds of diffractive optical elements

(a) Harmonic diffractive optical elements;

(b) single-layer diffractive optical elements

The HDOEs can realize the function of multiple wavebands imaging in the same position, in order to increase the operational wavebands of the refractive diffractive hybrid imaging optical system and ensure the imaging quality of the system. Generally, the machining errors of single point diamond turning on the HDOEs include the errors of diffractive microstructure cycle width and the errors of diffractive microstructure height. The effects can be expressed as shown in Figure 1.

The microstructure height errors of harmonic diffraction optical elements is shown in Figure 2 (a). $H_{\text {designed }}$ represents microstructure height obtained through theoretical calculation. $H_{\text {real }}$ represents the real microstructure height calculated through machining errors. The dotted line represents the theoretical diffractive microstructure, and the solid line represents the diffractive microstructure obtained by the actual machining. The schematic diagram of the cycle width errors is shown in Figure 
2(b). $T_{\text {designed }}$ represents the diffractive microstructure cycle width obtained through theoretical calculation. $T_{\text {real }}$ represents the real microstructure cycle width calculated through machining errors.

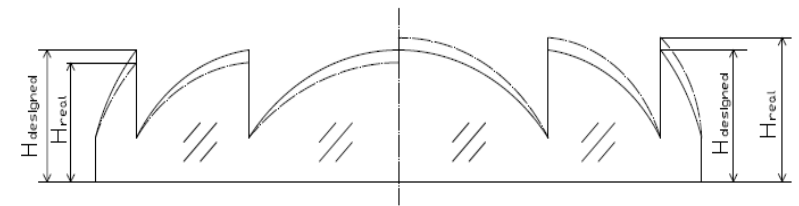

(a)

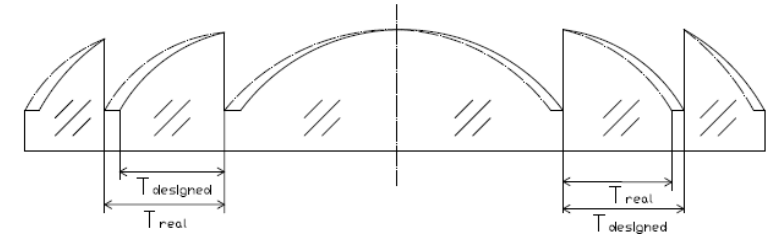

(b)

Fig. 2 The machining errors of the harmonic diffractive optical elements

(a)microstructure height errors;

(b)microstructure cycle width errors

In the refractive diffractive hybrid imaging optical system, the actual transfer function of the optical system is the product of the theoretical transfer function and polychromatic integral average diffraction efficiency of the diffraction optical elements. It can be shown as:

$$
M T F_{\text {poly }}=\bar{\eta}(\theta) \cdot \operatorname{MTF}_{\text {theo }}\left(f_{x}, f_{y}\right) \text {, }
$$

$\operatorname{MTF}_{\text {theo }}\left(f_{x}, f_{y}\right)$ is the theoretical optical transfer function of the refractive diffractive hybrid imaging optical system. $\bar{\eta}(\theta)$ is the polychromatic integral average diffraction efficiency in general incidence condition. When there is no machining errors, according to the scalar diffraction theory, the average diffraction efficiency of the polychromatic integral can be expressed as ${ }^{[13]}$ :

$$
\bar{\eta}(\theta)=\frac{1}{\lambda_{\max }-\lambda_{\min }} \int_{\lambda_{\min }}^{\lambda_{\max }} \eta_{m}^{\infty}(\lambda, \theta) d \lambda,
$$

$\lambda_{\min }$ and $\lambda_{\max }$ represents the minimum and maximum incident wavelength of optical elements. $\eta_{m}^{\infty}(\lambda, \theta)$ represents the diffraction efficiency when the incident wavelength $\lambda$ shoot into HDOEs in the angle of $\theta$. It can be expressed as ${ }^{[14]}$ :

$$
\eta_{m \text { designed }}^{\infty}(\lambda, \theta)=\sin c(m-\phi(\lambda, \theta)),
$$

$\phi(\lambda, \theta)$ represents the phrase. $m$ is the diffractive order. It can be seen that the diffraction efficiency can be $100 \%$ guaranteed when $m=\phi(\lambda, \theta)$. The phrase can be expressed as:

$$
\phi(\lambda, \theta)=\frac{H_{\text {designed }}}{\lambda}\left[\sqrt{n^{2}(\lambda)-n_{m}^{2}(\lambda) \sin \theta}-n(\lambda) \cos \theta\right],
$$

$H_{\text {designed }}$ is the design wavelength of the HDOEs. And $H_{\text {designed }}=\frac{p \cdot \lambda_{\text {designed }}}{n(\lambda)_{\text {designed }}-1} \cdot \theta$ is the incident angle. The cycle width is the result of the optical design software.

The machining errors of the HDOEs resulting from single point diamond turning are two kinds, including the cycle width errors and the height errors of the diffraction microstructure. They can be expressed as:

$$
\begin{gathered}
H_{\text {real }}=H_{\text {designed }} \pm \Delta H=H_{\text {designed }}(1 \pm \varepsilon), \\
T_{\text {real }}=T_{\text {designed }} \pm \Delta T=T_{\text {designed }}(1 \pm \xi),
\end{gathered}
$$


In the above formula, $\varepsilon$ and $\xi$ respectively represent the relative height errors of the diffractive microstructure and the relative cycle width errors. $\Delta H$ and $\Delta T$ respectively represent average height errors of the diffractive microstructure and the average cycle width errors. They can be expressed as:

$$
\begin{gathered}
\Delta H=\left(\Delta H_{1}+\Delta H_{2}+\cdots+\Delta H_{N}\right) / N, \\
\Delta T=\left(\Delta T_{1}+\Delta T_{2}+\cdots+\Delta T_{N}\right) / N,
\end{gathered}
$$

The formula (5) and formula (6) can be substituted into (3) to obtain the diffraction efficiency expression of the dual-waveband harmonic diffraction optical elements with machining errors being included. The formula goes as:

$$
\begin{aligned}
& \eta_{m \text { real }}^{\infty}(\lambda, \theta)=\sin c(m-\phi(\lambda, \theta)) \sin ^{2}(\xi) \\
& =\sin c\left(m-\frac{H_{\text {real }}}{\lambda}\left[\sqrt{n^{2}(\lambda)-n_{m}^{2}(\lambda) \sin \theta}-n(\lambda) \cos \theta\right]\right) \cdot \sin ^{2}(\xi)
\end{aligned}
$$

The formula (9) can be substituted into formula (2) to obtain the average diffraction efficiency expression of the polychromatic integral with machining errors being included. The formula goes as:

$$
\bar{\eta}(\theta)=\frac{1}{\lambda_{\max }-\lambda_{\min }} \int_{\lambda_{\min }}^{\lambda_{\max }} \eta_{m \text { real }}^{\infty}(\lambda, \theta) d \lambda .
$$

\section{Instance Analysis and Discussion}

Based on the effects of machining errors on PIDE, the machining error tolerance of dual-waveband harmonic diffractive optical elements has been analyzed. The height errors and cycle width errors of the diffractive microstructure have different sensitivity toward different waveband harmonic diffractive optical elements. They distribute machining errors between diffraction efficiency of the HDOEs and average diffraction efficiency of the polychromatic integral. Based on the error distribution, this paper analyzes the effects of the diffraction efficiency within the scope of the two wavebands under the circumstance of the two machining errors. The analysis results can be used for the machining error distribution of the harmonic diffraction optical elements in the refractive harmonic diffraction optical system. Therefore, the design of the optical system can be guided.

Firstly, the relationship among incident wavelength, incidence angle and diffraction efficiency of the dual-waveband harmonic diffractive optical elements without machining errors is simulated and the result is shown in Figure 3.

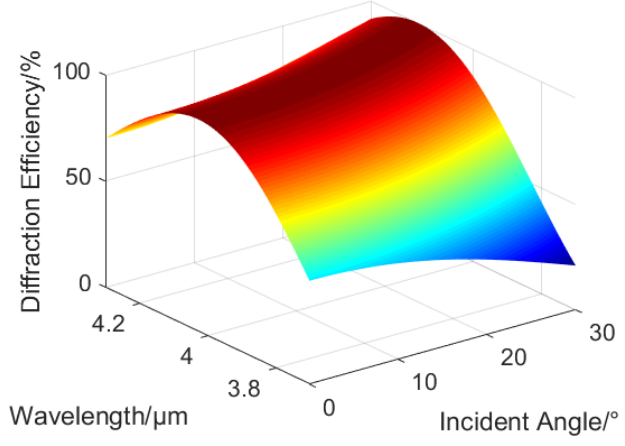

(a)

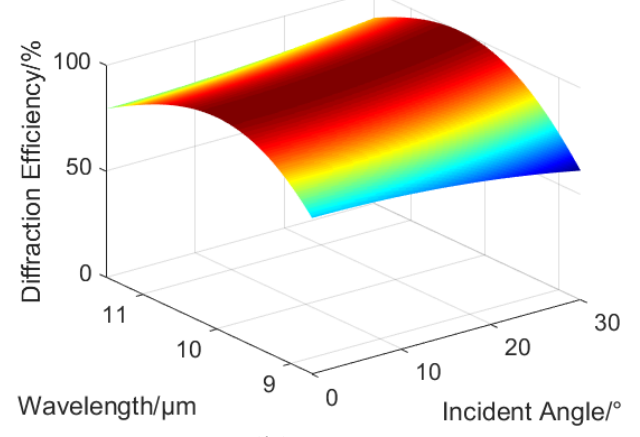

(b)

Fig.3 Relationship among wavelength-angle of incident-diffraction efficiency

(a. mid-waveband; b. long-waveband)

It can be seen that when there is no machining error, the diffraction efficiency of dual-waveband harmonic diffractive optical elements is insensitive to the incident angle. This is similar to diffraction efficiency characteristics of single-layer diffractive optical elements. It is good to improve the shortcoming that the diffraction efficiency of double-layer diffractive optical elements decreases fast with the incidence angles, which can be applied in dual-waveband infrared optical system with a wider incident angle. 
Then the effect of the machining errors on the average diffraction efficiency of the polychromatic integral is analyzed. Because the cycle width errors is a constant for the average diffraction efficiency of the polychromatic integral, the height of diffractive microstructure has greater effect on it, which is related to the integral wavelength. Next, the effect of the machining errors is analyzed under the condition of vertical incidence and normal incidence. In addition, based on the result, the machining errors will be distributed. After that, the effect on its diffraction efficiency will be calculated.

First of all, when the vertical incident light beam to the surface of dual-waveband harmonic diffractive optical elements, and the relative error of cycle width of diffraction microstructure is a given value, the effect of relative errors of the diffractive microstructure height on PIDE of dual-waveband harmonic diffractive optical elements is analyzed and the result is shown in Figure 4 and Table 1.

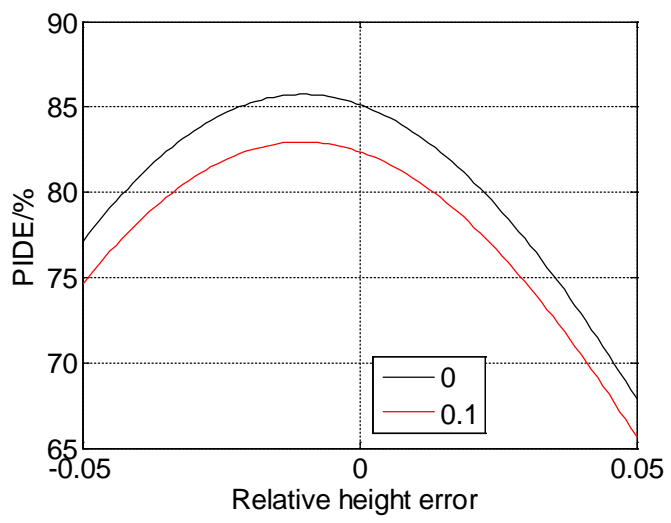

(a)

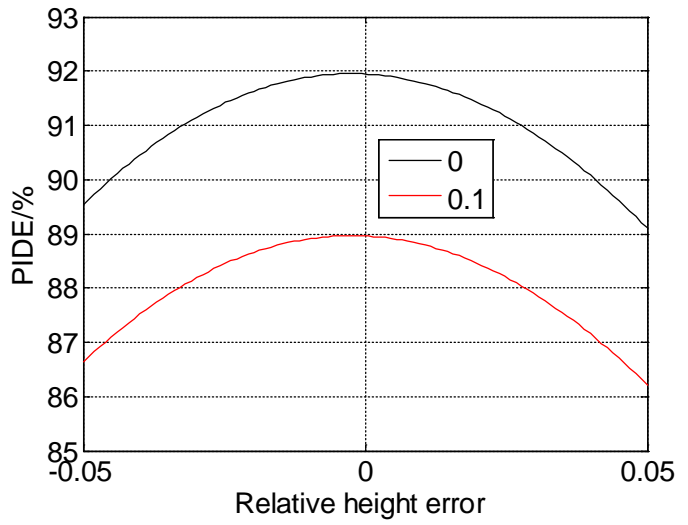

(b)

Fig.4 Machining errors effect on PIDE of HDOEs

(a. mid-waveband; b. long-waveband)

(b.

Tab1. PIDE for Machining errors

\begin{tabular}{ccc}
\hline $\begin{array}{c}\text { Relative height } \\
\text { errors }\end{array}$ & Middle-infrared waveband & Long-infrared waveband \\
\hline-0.1 & 74.600 & 86.632 \\
0 & 82.370 & 88.960 \\
0.1 & 65.650 & 86.215 \\
\hline
\end{tabular}

It can be seen that the machining errors have a certain effect on PIDE. The effect of the design waveband (long-infrared waveband) is smaller than that in the middle-infrared waveband.

When HDOEs has a certain incident Angle, the paper chooses the angle $15^{\circ}$ as an example and analyzes the effect of relative height errors on PIDE. The result is shown in Figure 8 and Table 2.

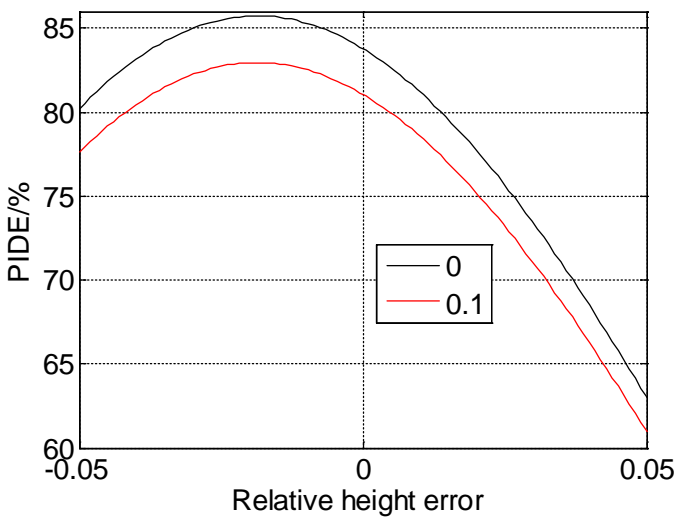

(a)

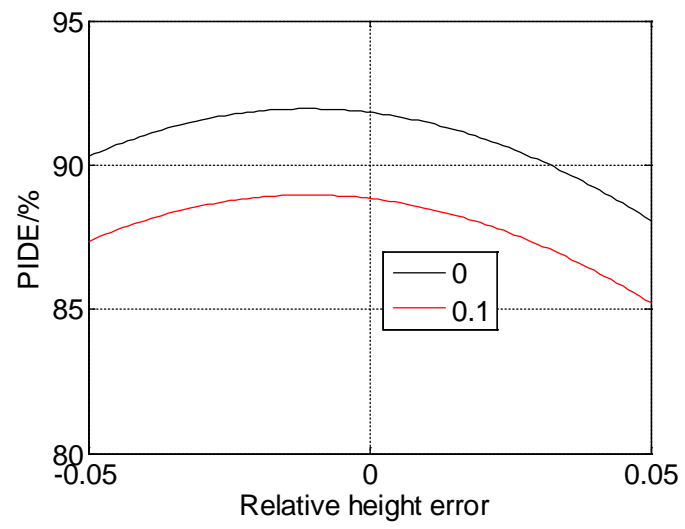

(b)

Fig.5 Machining errors effect on PIDE of HDOEs (a. mid-waveband; b. long-waveband) 
Tab2. PIDE in different cycle width

\begin{tabular}{ccc}
\hline $\begin{array}{c}\text { Relative height } \\
\text { errors }\end{array}$ & Middle-infrared waveband & Long-infrared waveband \\
\hline-0.1 & 77.593 & 87.360 \\
0 & 81.068 & 88.842 \\
0.1 & 60.952 & 85.221 \\
\hline
\end{tabular}

It can be seen from the analysis results that in the condition of oblique incidence, the diffraction microstructure machining error has a certain effect on PIDE of dual-waveband HDOEs, which is similar to vertical incidence. In addition, by comparing the effects happened in the conditions of vertical incidence and oblique incidence, it can be seen that dual-waveband HDOEs can be applied to dual-waveband infrared optical system with a wider incident angle. What' s more, the height errors of diffractive microstructure have a greater effect on PIDE.

When the cycle width error of the diffractive microstructure is 0.1 , the relative height errors of the diffractive microstructure is $0,0.05$ and -0.05 , the effect of relative height errors of the diffractive microstructure on diffraction efficiency is analyzed as in Figure 6.

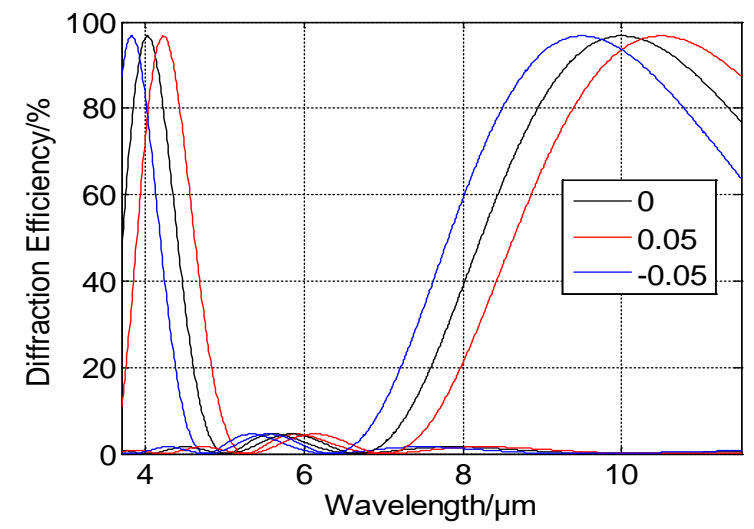

Fig.6 Relationship between microstructure width errors and diffraction efficiency

It can be seen that the height errors of the diffractive microstructure can reduce the diffraction efficiency at the position of the design wavelength. The corresponding wavelength of $100 \%$ diffraction efficiency will deviate from the design wavelength of dual-waveband HDOEs. When the relative height error is positive, $100 \%$ diffraction efficiency will shift to long wave. When the relative height error is negative, $100 \%$ diffraction efficiency will shift to short wave.

At last, when the cycle width errors of the diffractive microstructure is 0 and 0.1 , the relative height errors of the diffractive microstructure is $0,0.05$ and -0.05 , the effect on diffraction efficiency of dual-waveband HDOEs is analyzed as in Figure 7.

Figure 7(a) is the effect of cycle width when the error is 0.05 and Figure 7(b) is the effect when the error is -0.05 .

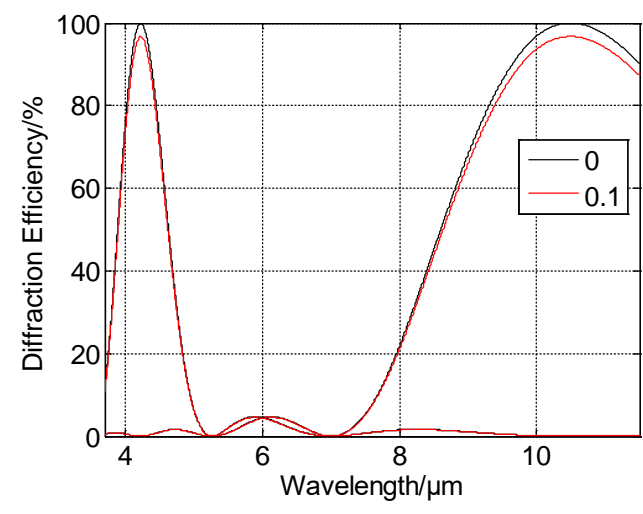

(a)

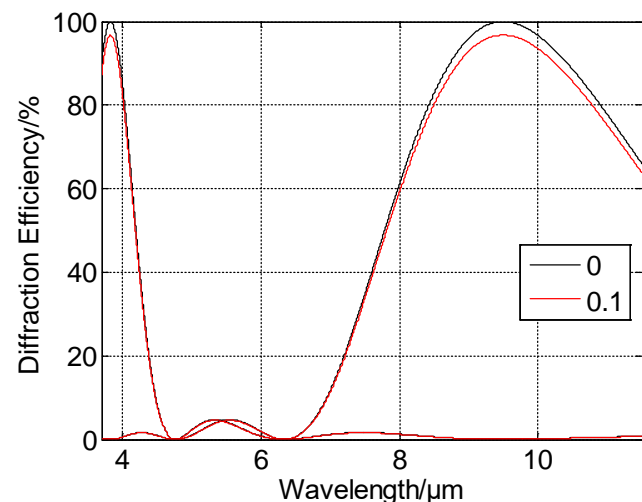

(b)

Fig.7 Relationship between microstructure height errors and diffraction efficiency(a.0.05; b.-0.05) 
It can be seen from the simulation results, under the circumstance of different relative height errors of diffractive microstructure, the effects of relative height errors on diffraction efficiency of dual-waveband HDOEs is greater than relative cycle width errors itself.

\section{Conclusion}

Based on PIDE expression of HDOEs, the model of effects of machining errors (height errors and cycle width errors) which is caused by single point diamond turning on dual-waveband HDOEs is established. The model can be used to determine the imaging quality of the diffractive hybrid imaging optical system. It can be seen that the effect of the incident angle on dual-waveband HDOEs is little. What' s more, when the relative height error of the diffractive microstructure is negative, the effect on PIDE is less. When there is vertical incidence and without machining errors, the PIDE of the medium waveband is $81.068 \%$, and the PIDE of the long waveband is $88.960 \%$. When the relative height error is -0.1, the PIDE of the 3.7-4.3um band is 74.600\% (decreased 7.770\%), and the PIDE of the long wave band is $86.632 \%$ (decreased $2.328 \%$ ). When the incident angle is $15^{\circ}$ and there is no machining errors, the PIDE of the medium waveband is $81.068 \%$, the PIDE of the long wave band is $88.842 \%$. When the relative height error is -0.1 , the PIDE of the medium waveband is $77.593 \%$ (decreased 3.475\%), and the PIDE of the long wave band is $87.360 \%$ (decreased $1.482 \%$ ). At last, based on the effects of machining errors on PIDE, the two machining errors have been distributed. The results can be applied to the design and machining error distribution of dual-band HDOEs.

\section{References}

[1] Dorodnyy A,Shklover V,Braginsky L,et al . High-efficiency spectrum splitting for solar photovoltaics[J]. Solar Energy Materials \& Solar Cells,2015,136(10): 120-126.

[2] Zhang W, Gong Y. Design of diffractive optical elements for off-axis illumination in projection lithography[J]. Opt. Precision Eng., 2008, 16(11):2081-2086. (in Chinese).

[3] Zhang Jian, Li Mengjuan, Yin Gang-hua, etal. Large-diameter membrane Fresnel diffraction elements for space telescope[J]. Optics and precision engineering, 2016, 24(6):1289-1296.

[4] Song Qiang, Zhu Jing, Wang Jian. A Mixed Gradient Algorithm for High Performance DOE Design in Off-Axis Lithography Illumination System[J]. Acta Optica Sinica, 2015,35(1):0122005-1-0122005-9. (in Chinese).

[5] Bao Hai-ting, Ouyang Mingzhao, Wang Zhiyong, Zhang Lina.. Design of Light Field Shaping System Based on DOE Identification[J]. Journal of Changchun University of Science and Technology, 2017,40(2): 27-32. (in Chinese).

[6] Liang Chuanyang, Zhang Wei, Rui Dawei, Sui Yngxin, Yang Huaijiang. High-performance laser projection display illumination system based on a diffractive optical element [J]. Applied Optics, 2017, 56(10):2810-2815.

[7] Bai Yu, Xing Tingwen, Jiang Yadong, Feng Cheng. Design of head-mounted display optical system with DOE[J]. Infrared and Laser Engineering, 2012,41(10): 2753-2758 (in Chinese).

[8]Liang Chuanyang, Zhang Wei, Wu Zhihui, Rui Dawei, Sui Yongxin, Yang Huaijiang. Beam shaping and speckle reduction in laser projection display systems using a vibrating diffractive optical element [J]. Current Optics and Photonics, 2017, 1(1):23-28.

[9] D. W. Sweeney, G. E. Sommargren. Harnonic diffractive lenses. Appl. Opt, 1995, 34(14):2469-2475.

[10] D. A. Burali and G. Michael Morris, "Effects of diffraction efficiency on the modulation transfer function of diffractive lenses,” Appl. Opt. 1992,31(10), 4389 - 4396. 
[11] L. Yang, Q. Cui, T. Liu, and C. Xue, "Effects of machining errors on diffraction efficiency for multilayer diffractive optical elements,” Appl. Opt.2011,50(18), 6128 - 6133 .

[12] Shan Mao,Qingfeng Cui,Mingxu Piao. Tolerance analysis of multilayer diffractive optics based on polychromatic integral diffraction efficiency. Appl. Opt.2015, 54(36), 032707 - 035702.

[13] Cui qing-feng. Design of hybrid diffractive-refractive imaging optical systems[J]. Infrared and Laser Engineering, 2006,35(1): 12-16. (in Chinese).

[14] D. C. O’ Shea, T. J. Suleski, A. D. Kathman, and D. W. Prather, Diffractive Optics Design, Fabrication, and Test (SPIE, 2004). 